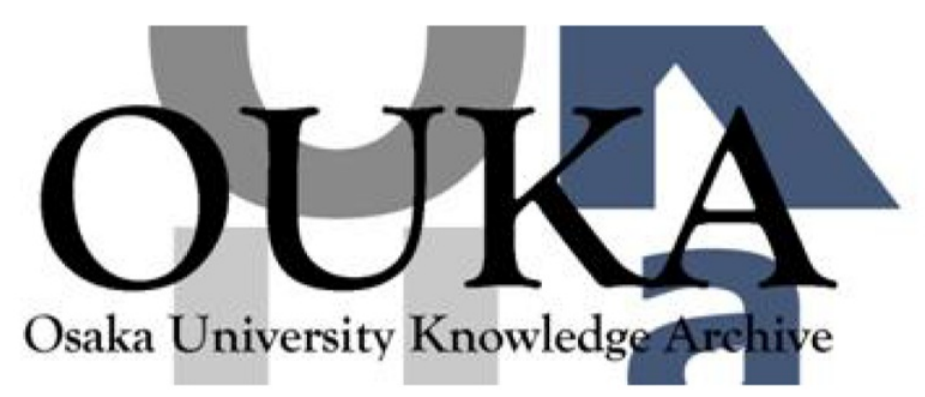

\begin{tabular}{|c|l|}
\hline Title & $\begin{array}{l}\text { Dimensions and viscosity behavior of } \\
\text { polyelectrolyte brushes in aqueous sodium } \\
\text { chloride. A polymacromonomer consisting of } \\
\text { sodium poly(styrene sulfonate) }\end{array}$ \\
\hline Author(s) & Kanemaru, Eiji; Nakamura, Yo; Terao, Ken et al. \\
\hline Citation & Polymer. 49(19) p. 4174-p. 4179 \\
\hline Issue Date & $2008-09-09$ \\
\hline oaire:version & AM \\
\hline URL & https://hdl. handle.net/11094/25936 \\
\hline rights & \\
\hline Note & \\
\hline
\end{tabular}

Osaka University Knowledge Archive : OUKA

https://ir. Library. osaka-u. ac. jp/

Osaka University 


\title{
Dimensions and viscosity behavior of polyelectrolyte brushes in aqueous sodium chloride. A polymacromonomer consisting of sodium poly(styrene sulfonate)
}

\author{
Eiji Kanemaru ${ }^{a}$, Ken Terao ${ }^{\mathrm{a}, *}$, Yo Nakamura ${ }^{\mathrm{b}}$, Takashi Norisuye ${ }^{\mathrm{a}}$ \\ a Department of Macromolecular Science, Graduate School of Science, Osaka University, \\ 1-1, Machikaneyama-cho, Toyonaka, Osaka 560-0043, Japan \\ ${ }^{\mathrm{b}}$ Department of Polymer Chemistry, Graduate School of Engineering, Kyoto University, \\ Katsura, Nishikyo-ku, Kyoto 615-8510, Japan
}

\begin{abstract}
Nine samples of a polymacromonomer consisting of sodium poly(styrene sulfonate) and having a side-chain polymerization degree of 15 are investigated by light scattering, small-angle $\mathrm{X}$-ray scattering, and viscometry with $0.05 \mathrm{M}$ aqueous $\mathrm{NaCl}$ at $25^{\circ} \mathrm{C}$ as the solvent. The (total) weight-average molecular weight $M_{\mathrm{w}}$ ranges from $2.2 \times 10^{4}$ to $7.1 \times$ $10^{6}$. The radii of gyration, scattering functions, and intrinsic viscosities determined as functions of $M_{\mathrm{w}}$ are analyzed in terms of the cylindrical wormlike chain model. The estimated Kuhn segment length of about $120 \mathrm{~nm}$ is much larger than that (16 nm) for the polystyrene polymacromonomer with the equivalent side-chain length in toluene, a good solvent, while the chain thickness $(5.5 \mathrm{~nm})$ is comparable to or only slightly larger than that $(5 \mathrm{~nm})$ of the nonionic polymer. It is thus concluded that the electrostatic repulsion between side chains significantly stiffens the main chain of the polyelectrolyte brush.
\end{abstract}

Keywords: Polyelectrolyte brush; Light scattering; Chain stiffness

* To whom correspondence should be addressed. Tel: +81 66850 5459; fax: +81 66850 5461; e-mail: kterao@chem.sci.osaka-u.ac.jp 


\section{Introduction}

Several decades ago, a few groups [1-4] showed that the main chains of some comb-like polymers in the theta solvent are more extended than those of the corresponding linear chains due to the high segment density near the main chain. This chain extension becomes more pronounced with increasing side-chain length and shortening side-chain spacing on the main chain [5], and comb polymers at the limit of the shortest spacing, i.e., so-called polymacromonomers [6,7] or cylindrical brushes, behave as semiflexible chains with backbone stiffness higher for a longer side chain [8-20]. Furthermore, their stiffness is higher in good solvents than in theta solvents [14-20], indicating that interactions between or among side chains are also responsible for stiffening the comb backbone [21]. Thus it is intriguing and probably significant to investigate the effect of electrostatic repulsion on the backbone stiffness of a polyelectrolyte brush having an ionized group on each side-chain monomer unit, though the effective charge density may be much lower than what is expected for the corresponding linear polyelectrolyte [22]. As far as we know, no experimental work has as yet been reported on the stiffness of an ionic polymacromonomer.

In the present work, we made light and small-angle X-ray scattering and viscosity measurements on a series of samples of a polymacromonomer consisting of sodium poly(styrene sulfonate) (Figure 1) in $0.05 \mathrm{M}$ aqueous sodium chloride to estimate the backbone stiffness of the polyelectrolyte brush. We prepared those samples by sulfonation of the styrene units of polystyrene polymacromonomer samples [14] with different main-chain lengths. The use of such polyelectrolyte samples has two advantages: (1) The dilute-solution behavior of each side chain, i.e., sodium poly(styrene sulfonate), has extensively been studied [23-26] and thus fairly well understood, and (2) the direct sulfonation should allow us to compare solution properties of the ionic and nonionic polymacromonomers having the exactly equivalent side-chain length. The radii of gyration, particle scattering functions, and intrinsic viscosities obtained for polyeletrolyte brush samples are analyzed below in terms of the wormlike chain [27].

\section{Experimental section}

\subsection{Samples}

The previously investigated polymacromonomer F15 samples [14] (F15-1, F15-2, ... F15-5, F15-11, F15-12 ... F15-14) having 15 styrene units on each side chain were sulfonated by Vink's method [28]. The typical procedure was as follows: A cyclohexane solution $\left(70 \mathrm{~cm}^{3}\right)$ of each $\mathrm{F} 15$ sample was mixed with $40 \mathrm{~cm}^{3}$ of concentrated 
sulfuric acid containing $7 \mathrm{~g}$ of $\mathrm{P}_{2} \mathrm{O}_{5}$ as a drying agent, followed by stirring for 2-3 hours at $40{ }^{\circ} \mathrm{C}$, and water was added to it at $0{ }^{\circ} \mathrm{C}$. The water phase was extracted from the mixture, dialyzed against pure water for seven days, and mixed with a large amount of 0.5 $\mathrm{M}$ aqueous $\mathrm{NaOH}$. The solution was then subjected to fractional precipitation (see Ref. $[23,24])$ for the purpose of purifying the product. An appropriate middle fraction was passed through an ion exchanger column (after being dissolved in deionized water), neutralized, and freeze-dried in the manner employed for Na poly(styrene sulfonate) [24].

The polymacromonomer samples (SF15) consisting of $\mathrm{Na}$ poly(styrene sulfonate) prepared and purified in this way were designated as SF15-1, SF15-2, ... SF15-9 in the order of descending molecular weight. The mass ratio of carbon to sulfate determined by elemental analysis indicated that these samples were almost fully sulfonated.

\subsection{Light scattering}

Scattering intensities for eight samples (SF15-1, SF15-2, ... SF15-8) of polymacromonomer SF15 in $0.05 \mathrm{M}$ aqueous $\mathrm{NaCl}$ at $25{ }^{\circ} \mathrm{C}$ were measured on a Fica-50 light scattering photometer with vertically polarized incident light of 436 or $546 \mathrm{~nm}$ wavelength $\left(\lambda_{0}\right)$. The experimental procedures were essentially the same as those described previously $[13,20,26]$. The weight-average molecular weight $M_{\mathrm{w}}$, the second virial coefficient $A_{2}$, and the $z$-average mean-square radius of gyration $\left\langle S^{2}\right\rangle_{z}$ were determined by the square-root plots [29] of $\left(K c / R_{\theta}\right)^{1 / 2}$ vs $\sin ^{2}(\theta / 2)$ and vs $c$ (the polymer mass concentration), where $K$ and $R_{\theta}$ denote the optical constant and the reduced scattering intensity at scattering angle $\theta$, respectively. Optical anisotropy effects on $M_{\mathrm{w}}, A_{2}$, and $\left.<S^{2}\right\rangle_{\mathrm{z}}$ were negligible for the samples studied.

The specific refractive index increments $(\partial n / \partial c)_{\mu}$ at fixed chemical potentials $\mu$ of diffusible components and at $25^{\circ} \mathrm{C}$ were measured for $0.05 \mathrm{M}$ aqueous $\mathrm{NaCl}$ solutions of sample SF15-7 dialyzed against the solvent (see Ref. [30] for the experimental details). The results of $(\partial \mathrm{n} / \partial c)_{\mu}$ were 0.176 and $0.168 \mathrm{~cm}^{3} \mathrm{~g}^{-1}$ at 436 and $546 \mathrm{~nm}$, respectively.

\subsection{Small-angle $X$-ray scattering (SAXS)}

SAXS intensities $I_{\theta}$ at $\theta$ were measured for the three lowest molecular weight samples SF15-7, SF15-8, and SF15-9 in 0.05M aqueous $\mathrm{NaCl}$ at $25^{\circ} \mathrm{C}$ on a Rigaku R-AXIS IV++ imaging plate at the BL40B2 beamline in SPring-8. Each test solution was filled in a 1.5 $\mathrm{mm} \phi$ quartz capillary. The wavelength, the camera length, and the accumulation time were set to be $0.10 \mathrm{~nm}, 1580 \mathrm{~mm}$, and $300 \mathrm{sec}$, respectively. The scattering angle at 
each pixel on the imaging plate was determined from the Bragg reflection of powdery lead stearate. The excess scattering intensity $\Delta I_{\theta}$ was obtained as the difference in $I_{\theta}$ between the solution and the solvent. Its concentration dependence and angular dependence were analyzed by use of the plots of $\left(c / \Delta I_{\theta}\right)^{1 / 2}$ vs $c$ and $\ln \left(c / \Delta I_{\theta}\right)$ vs $\sin ^{2}(\theta / 2)$, respectively, and the particle scattering function $P(\theta)$ was obtained as a function of $\theta$ or $k$ (the magnitude of the scattering vector) from the ratio of $\left(c / \Delta I_{0}\right)$ to $\left(c / \Delta I_{\theta}\right)$ both at infinite dilution.

\subsection{Sedimentation equilibrium}

Sedimentation equilibrium measurements were made on $0.05 \mathrm{M}$ aqueous $\mathrm{NaCl}$ solutions of the lowest molar mass sample SF15-9 at $25{ }^{\circ} \mathrm{C}$ in a Beckman Optima XL-1 ultracentrifuge to determine $M_{\mathrm{w}}, A_{2}$, and the z-average molecular weight $M_{\mathrm{z}}$ (see Ref. [31] for the procedure of data analysis). A 12-mm double-sector cell was used and the rotor speed was chosen to be $20000 \mathrm{rpm}$. The concentration profile was obtained from each interference pattern with a diode laser of $\lambda_{0}=675 \mathrm{~nm}$. The $(\partial n / \partial c)_{\mu}$ at this wavelength was estimated to be $0.163 \mathrm{~cm}^{3} \mathrm{~g}^{-1}$ from the data at 436 and $546 \mathrm{~nm}$ with the aid of the $(\partial n / \partial c)_{\mu}$ vs $\lambda_{0}{ }^{-2}$ plot. The density increment $(\partial \rho / \partial c)_{\mu}$ at fixed $\mu$ was determined for SF15-7 in $0.05 \mathrm{M}$ aqueous $\mathrm{NaCl}$ at $25{ }^{\circ} \mathrm{C}$ to be 0.374 using an Anton Paar DMA 5000 densitometer.

\subsection{Viscometry}

Zero-shear rate intrinsic viscosities $[\eta]$ in $0.05 \mathrm{M}$ and $0.005 \mathrm{M}$ aqueous $\mathrm{NaCl}$ were determined at $25{ }^{\circ} \mathrm{C}$ using a four-bulb low-shear viscometer of the Ubbelohde type for higher molecular weight samples SF15-1, SF15-2, SF15-3, and SF15-5 and capillary viscometers of the Ubbelohde type for the rest; some samples (SF15-4 at $0.05 \mathrm{M} \mathrm{NaCl}$ and SF15-3 - SF15-6 at 0.005 M NaCl) were not studied because of their limited quantities. In evaluation of the relative viscosity $\eta_{\mathrm{r}}$, the difference between the solution and solvent densities was taken into account.

\section{Results}

\subsection{Dimensional properties}

Figure 2 illustrates the angular dependence of $\left(K c / R_{\theta}\right)_{c=0}^{1 / 2}$, i.e., the infinite dilution value of $\left(K c / R_{\theta}\right)^{1 / 2}$, for samples SF15-1, SF15-2, ... SF15-5 in 0.05 M aqueous $\mathrm{NaCl}$. The intercepts and initial slopes of the indicated curves (the dashed lines) give $M_{\mathrm{w}}$ and $<S^{2}>_{\mathrm{z}}$ for these samples. 
Numerical results from light scattering measurements are summarized in Table I, along with those from sedimentation equilibrium. The $M_{\mathrm{w}}$ value for SF15-1 is about $40 \%$ smaller than the expected value, which is estimated to be 1.93 times as large as $M_{\mathrm{w}}$ of the precursor (i.e., polystyrene polymacromonomer F15). For the other SF15 samples, however, the difference between the expected and observed $M_{\mathrm{w}}$ 's is less than $\pm 25 \%$. Such a small difference is most likely due to the fractional precipitation mentioned in the Experimental section.

Figure 3 shows the $k^{2}$-dependence of $P(\theta)$ from SAXS in the form of the Guinier plot for SF15-7, SF15-8, and SF15-9 in $0.05 \mathrm{M}$ aqueous $\mathrm{NaCl}$. The radii of gyration evaluated from the indicated straight lines (the dashed line for SF15-7) are presented in Table I.

The $\left\langle S^{2}\right\rangle_{\mathrm{z}}$ data for SF15 samples in the aqueous salt at $25^{\circ} \mathrm{C}$ (the circles) are plotted against $N_{\mathrm{w}}$ (the weight-average number of main-chain residues) in Figure 4, along with our previous data [14,32,33] for polystyrene polymacromonomer $\mathrm{F} 15$ in toluene at $15{ }^{\circ} \mathrm{C}$ (a good solvent, the triangles) and cyclohexane at $34.5{ }^{\circ} \mathrm{C}$ (a theta solvent, the squares). Here, $N_{\mathrm{w}}$ has been calculated from $M_{\mathrm{w}} / M_{0}$ with $M_{0}$ (the weight-average molecular weight of the macromonomer unit) $=3.18 \times 10^{3}$ for SF15 and $1.65 \times 10^{3}$ for F15 [14]. When compared at the same $N_{\mathrm{w}},\left\langle S^{2}\right\rangle_{\mathrm{z}}^{1 / 2}$ for SF15 is much larger than those for F15 in the two organic solvents for $N_{\mathrm{w}}>4 \times 10^{2}$, the difference being two to three times at $N_{\mathrm{w}}=2000$. As $N_{\mathrm{w}}$ decreases below 50 , the dimensions of the ionic and nonionic polymacromonomers come close to one another. Since the latter polymer has a higher stiffness in the good solvent than in the theta solvent, the largest $\left\langle S^{2}\right\rangle_{\mathrm{z}}{ }^{1 / 2}$ of the former polymer suggests the highest stiffness of the chain among the three systems.

\subsection{Viscosity behavior}

The fifth and sixth columns of Table I give data for $[\eta]$ and $k^{\prime}$ (Huggins' constant), respectively, where the bracketed values refer to $0.005 \mathrm{M}$ aqueous $\mathrm{NaCl}$ at $25{ }^{\circ} \mathrm{C}$. As expected for polyelectrolytes, $[\eta]$ is larger at this lower salt concentration than at $0.05 \mathrm{M}$. In connection with $[\eta]$ for low molecular weight samples, some remarks are pertinent here.

Figures 5(a) and 5(b) show the $\left(\ln \eta_{\mathrm{r}}\right) / c$ vs $c$ plots for the four lowest molecular weight samples in 0.005 and $0.05 \mathrm{M}$ aqueous $\mathrm{NaCl}$, respectively. In the former figure, the curves remarkably bend down, revealing viscosity behavior typical of polyelectrolytes at low ionic strength. This remarkable bending does not allow the determination of $[\eta]$. In the latter figure, each curve still has a positive slope but less pronounced curvature, thus giving 
$[\eta]$ with moderate accuracy. These features of the viscosity plots are similar to what is known for $\mathrm{Na}$ poly(styrene sulfonate) of low molecular weight in the aqueous salts [25].

Figure 6 illustrates the $N_{\mathrm{w}}$-dependence of [ $\left.\eta\right] M_{0}$ for the SF15 polyelectrolyte in $0.05 \mathrm{M}$ aqueous $\mathrm{NaCl}$ (the unfilled circles) in comparison with that for the F15 polymer in good (toluene) and theta (cyclohexane) solvents [15]; the filled circles represent the data in $0.005 \mathrm{M}$ aqueous $\mathrm{NaCl}$. The value of $[\eta]$ for SF15 in $0.05 \mathrm{M}$ aqueous $\mathrm{NaCl}$ is much larger than those for F15 at the same $N_{\mathrm{w}}$ above $10^{3}$. This is consistent with the $\left\langle S^{2}\right\rangle_{\mathrm{z}}$ data in Figure 4, supporting the suggestion from $\left\langle S^{2}\right\rangle_{z}$ that the backbone stiffness of the polyelectrolyte brush should be the highest among the three systems. As $N_{\mathrm{w}}$ lowers, $[\eta]$ for SF15 sharply decreases, becomes almost independent of $N_{\mathrm{w}}$, and finally increases. This upswing of [ $\eta]$ at low $N_{\mathrm{w}}$ is explained in the next section.

\section{Discussion}

\subsection{Analysis of scattering data and molecular characteristics}

We analyze the present scattering data for the SF15 polymacromonomer in $0.05 \mathrm{M}$ aqueous $\mathrm{NaCl}$ using available theories $[34,35]$ for the cylindrical wormlike chain. This model has successfully been applied to determine the molecular characteristics of nonionic polymacromonomers in both theta and good solvents [10-20]. Its mean-square radius of gyration $<S^{2}>$ is expressed by $[34,36]$

$$
\left\langle S^{2}\right\rangle=\frac{L}{6 \lambda}-\frac{1}{4 \lambda^{2}}+\frac{1}{4 \lambda^{3} L}-\frac{1}{8 \lambda^{4} L^{2}}[1-\exp (-2 \lambda L)]+\frac{d^{2}}{8}
$$

where $L$ is the contour length, $\lambda^{-1}$ is the Kuhn segment length, and $d$ is the cylinder diameter. The first parameter is defined by the ratio of the molecular weight to the molar mass per unit contour length $M_{\mathrm{L}}$, but we write it as [15]

$$
L=\frac{M_{\mathrm{w}}}{M_{\mathrm{L}}}+\delta
$$

by taking into account the apparent contribution $\delta$ from side chains (near the main-chain ends) to $L$. Thus, eq 1 contains the four parameters $M_{\mathrm{L}}, \lambda^{-1}, d$, and $\delta$.

We estimated them from the $\left\langle S^{2}\right\rangle_{\mathrm{z}}$ data by curve fitting to be $M_{\mathrm{L}}=12000 \pm 2000 \mathrm{~nm}^{-1}$, $\lambda^{-1}=130 \pm 40 \mathrm{~nm}, d=5.5 \pm 0.5 \mathrm{~nm}$, and $\delta=1.5 \pm 1.5 \mathrm{~nm}$. The first two parameters 
were substantially determined only by the data for $M_{\mathrm{w}}>10^{6}$, and thus the fitting to all the data points allowed the unique determination of the four parameters though the uncertainty of $\delta$ was large. Figure 7(a) shows that the molecular weight dependence of $\left\langle S^{2}\right\rangle_{\mathrm{z}}^{1 / 2}$ for the polyelectrolyte brush is accurately described by eq 1 with $M_{\mathrm{L}}=12000 \mathrm{~nm}^{-1}, \lambda^{-1}=130$ $\mathrm{nm}, d=5.5 \mathrm{~nm}$, and $\delta=1.5 \mathrm{~nm}$. Importantly, this $\lambda^{-1}$ value of $130 \mathrm{~nm}$ is about 8 and 14 times larger than those for the polystyrene polymacromonomer F15 in toluene and cyclohexane, respectively, demonstrating that sulfonation remarkably stiffens the backbone of the polymer. The estimated $M_{\mathrm{L}}$ yields $0.27( \pm 0.05) \mathrm{nm}$ for the contour length per repeat unit $h\left(\equiv M_{0} / M_{\mathrm{L}}\right)$. This $h$ is close to the value $(0.25 \mathrm{~nm})$ expected for the trans-planer conformation of the main chain and also to those $(0.26-0.27 \mathrm{~nm})$ estimated from $\left\langle S^{2}\right\rangle_{\mathrm{z}}$ and $[\eta]$ for the F15 polymacromonomer in toluene and cyclohexane $[14,15]$. The diameter of the SF15 brush $(5.5 \pm 0.5 \mathrm{~nm})$ is also comparable to or only slightly larger than those of F15 in toluene $(5 \mathrm{~nm})$ and cyclohexane $(4.7 \mathrm{~nm})$ [15,32,33]. We confirmed this diameter of SF15 by the cross-section plot, i.e., the $\ln [k P(\theta)]$ vs $k^{2}$ plot, for the present SAXS data, which gave essentially the same $d$ of $6.0 \mathrm{~nm}$. It should be noted that the $d$ of $5.5-6.0 \mathrm{~nm}$ is considerably smaller than the fully stretched value of $7.5 \mathrm{~nm}$ for SF15 (= $0.25 \mathrm{~nm} \times 15 \times 2)$.

In the above analysis of $\left\langle S^{2}\right\rangle_{\mathrm{z}}$, no excluded-volume effect was considered. According to our previous estimates [14,15], this effect in toluene solutions of the F15 polymacromonomer becomes appreciable when the Kuhn segment number $\lambda L$ exceeds 5 6. This critical value is comparable to the Kuhn segment number $5( \pm 3)$ for our highest $M_{\mathrm{w}}$ sample. This coincidence appears to suggest negligible excluded-volume effects on $\left\langle S^{2}\right\rangle_{\mathrm{z}}$ for all the samples studied here, but a closer examination is necessary because excluded-volume interactions must be much stronger for the ionic SF15 polymer in $0.05 \mathrm{M}$ aqueous $\mathrm{NaCl}$ than for the nonionic F15 polymer in toluene. In the framework of the Yamakawa-Stockmayer-Shimada theory [37-39] for wormlike or helical-wormlike bead chains [39], the radius expansion factor (= the ratio of perturbed to unperturbed $\left\langle S^{2}\right\rangle^{1 / 2}$ ) is a universal function of the scaled excluded-volume parameter. For the two polymacromonomers with the same $\lambda L$, this parameter differs only in $\lambda \beta_{2, \mathrm{~b}}$ (i.e., the binary cluster integral for the bead - bead interaction relative to $\lambda^{-1}$ ), and $\beta_{2, \mathrm{~b}}$ may be taken to equal the product of a constant $C$ and the binary cluster integral $\beta_{2}$ for the side chain units, i.e., for the monomeric units of Na poly(styrene sulfonate) or polystyrene; the constant $C$ must be the same for the two polymacromonomers consisting of 15 side-chain residues and need not be specified for here. Using $\beta_{2}=0.25 \mathrm{~nm}^{3}$ for Na poly(styrene sulfonate) in 
the aqueous salt $[25,26]$ and $\beta_{2}=0.033 \mathrm{~nm}^{3}$ for polystyrene in the organic solvent [39], we get $\lambda \beta_{2, \mathrm{~b}}=1.9 \times 10^{-3} \mathrm{C}$ and $2.1 \times 10^{-3} \mathrm{C} \mathrm{nm^{2 }}$ for the SF15 and F15 systems, respectively. These $\lambda \beta_{2, \mathrm{~b}}$ 's, quite close to each other, indicate that, when $\lambda L$ is the same, the two polymer-solvent systems undergo roughly the same degrees of chain expansion. Hence, we may conclude that our polyelectrolyte brush suffers no significant intramolecular excluded-volume effect in the $M_{\mathrm{w}}$ range concerned.

Figure 8 compares the scattering functions (in the form of Kratky's plot) for samples SF15-7, SF15-8, and SF15-9 in 0.05 M aqueous $\mathrm{NaCl}$ with the theoretical curves for the wormlike cylinders computed from Nakamura and Norisuye's theory [35] with the set of parameters from $\left\langle S^{2}>_{\mathrm{z}}^{1 / 2}\left(M_{\mathrm{L}}=12000 \mathrm{~nm}^{-1}, \lambda^{-1}=130 \mathrm{~nm}, d=5.5 \mathrm{~nm}\right.\right.$, and $\left.\delta=1.5 \mathrm{~nm}\right)$. The theory quantitatively reproduces the experimental curves for SF15-7 and SF15-8 having large peaks around $k=0.5-0.6 \mathrm{~nm}^{-1}$, confirming the model parameters estimated from $\left\langle S^{2}>_{\mathrm{z}}^{1 / 2}\right.$. The downward deviation of the curve for sample SF15-9 at $k>1 \mathrm{~nm}^{-1}$ indicates that the cylinder model is too crude for the small number of side chains (only 7) at high scattering angles where X-ray sees the relatively local structure of the molecule. Similar behavior was observed for the corresponding F15 sample in cyclohexane [32] and toluene [33].

\subsection{Analysis of viscosity data}

The Yamakawa-Yoshizaki theory [40] for $[\eta]$ of a wormlike spherocylinder may be written as

$$
[\eta]=\frac{f(\lambda L, \lambda d)}{\lambda^{3} M}
$$

where $M$ denotes the molecular weight of the polymer and $f(\lambda L, \lambda d)$ is a known function of $\lambda L$ and $\lambda d$. The four parameters $M_{\mathrm{L}}, \lambda^{-1}, d$, and $\delta$ cannot uniquely be determined from the present $[\eta]$ data. When $d$ is assumed to be $5.5 \mathrm{~nm}$ obtained from $\left\langle S^{2}\right\rangle_{\mathrm{z}}{ }^{1 / 2}$, the best fit to the experimental data was found for $M_{\mathrm{L}}=14000 \mathrm{~nm}^{-1}, \lambda^{-1}=110 \mathrm{~nm}$, and $\delta=4.3 \mathrm{~nm}$ by curve fitting, as shown in Figure 7(b). In actuality, however, the following parameter range led to substantially the same theoretical curves in the $M_{\mathrm{w}}$ range of our SF15 samples: $\pm 1000 \mathrm{~nm}^{-1}$ for $M_{\mathrm{L}}, \pm 30 \mathrm{~nm}$ for $\lambda^{-1}$, and $\pm 0.4 \mathrm{~nm}$ for $\delta$. The $M_{\mathrm{L}}$ of $14000 \mathrm{~nm}^{-1}$ gives $h$ a value of $0.23 \mathrm{~nm}$, which does not differ much from $0.25 \mathrm{~nm}$ for the all trans conformation. The estimated stiffness is also close to that from $\left\langle S^{2}\right\rangle_{\mathrm{z}}^{1 / 2}$. Thus, we may conclude that the 
available theories for the wormlike cylinder consistently explain the data of $\left\langle S^{2}\right\rangle_{\mathrm{z}}, P(\theta)$, and $[\eta]$ for the SF15 polyelectrolyte brush in $0.05 \mathrm{M}$ aqueous $\mathrm{NaCl}$.

We discuss the observed minimum in the $\log [\eta]$ vs $\log M_{\mathrm{w}}$ relation (Figure 6 or 7 ) following the recent interpretation by Sugiyama et al. [20] for a polystyrene polymacromonomer with 113 units in each side chain and confining ourselves to the rod limit of the wormlike spherocylinder. Taking into account the effect of $\delta$ (eq 2), we have in this limit [41]

$$
[\eta] \propto \frac{d^{2} p^{3}}{p-(\delta / d)} F(p) \quad \text { (rod limit) }
$$

where $p$ is the axis ratio defined by $L / d$ and $F(p)$ is a known function of $p$. As $p$ decreases with both $d$ and $\delta$ fixed, $p^{3} F(p)$ decreases while the factor $(p-\delta / d)^{-1}$ increases. As the result, $[\eta]$ has a broad minimum around $p=2.3 \delta / d$ (for $\delta / d=0.78$ ), which corresponds to $N_{\mathrm{w}}=22$ or $M_{\mathrm{w}}=7 \times 10^{4}$. Since the upswing of $[\eta]$ with lowering $N_{\mathrm{w}}$ or $M_{\mathrm{w}}$ arises from the factor $(p-\delta / d)^{-1}$, the effect of $\delta$ on $[\eta]$ is significant at low molecular weights. In Figure 6, minima of $[\eta]$ 's for the F15 polymacromonomer in the two organic solvents can hardly be seen (though there exist very shallow minima around $N_{\mathrm{w}}=15$ ). It can be shown by use of eq 4 that this viscosity behavior, seemingly contrasted to that of SF15, is due to the smaller $\delta / d$ (about 0.46 ) of the polystyrene polymacromonomer.

\subsection{Main-chain stiffness}

The backbone stiffness of the polyelectrolyte brush SF15 expressed in terms of the mean $(120 \mathrm{~nm})$ of the $\lambda^{-1}$ values from $\left\langle S^{2}>_{\mathrm{z}}{ }^{1 / 2}\right.$ and $[\eta]$ is 7.5 times higher than that (16 nm) of the polystyrene polymacromonomer F15 with the same side-chain degree of polymerization in toluene. This is due primarily to enhanced side chain - side chain interactions in the SF15 polymer. In other words, sulfonation of F15 greatly stiffens the polymacromonomer backbone, and electrostatic repulsions between charged groups of the resultant polyelectrolyte are responsible for the very high stiffness.

According to Nakamura and Norisuye's theory [21], $\lambda^{-1}$ is the sum of $\lambda_{b}{ }^{-1}$ (the increase of $\lambda^{-1}$ arising from side chain - side chain interactions) and $\lambda_{0}^{-1}$ (the contribution of the intrinsic stiffness) and the former is given by $\lambda_{\mathrm{b}}{ }^{-1}=(1 / 8 \pi)\left(n^{2} / h\right) \beta_{2}$, where $n$ stands for the polymerization degree of each side chain; we note that the contribution from main chain side chain interactions to $\lambda_{\mathrm{b}}{ }^{-1}$ is proportional to $n$ and ignored here. Using $\beta_{2}=0.25 \mathrm{~nm}^{3}$ 
for $\mathrm{Na}$ poly(styrene sulfonate) in $0.05 \mathrm{M}$ aqueous $\mathrm{NaCl}[25,26]$ and $h=0.25 \mathrm{~nm}$, we obtain $\lambda_{\mathrm{b}}{ }^{-1}=36 \mathrm{~nm}$, which is much smaller than the estimated $\lambda^{-1}$ of $120 \mathrm{~nm}$ even though $\lambda_{0}{ }^{-1}$ must be added to $\lambda_{\mathrm{b}}{ }^{-1} ; \lambda_{0}{ }^{-1}$ for the polyelectrolyte brush with the bulky sulfonated groups should be larger than that $(12 \mathrm{~nm})$ often chosen for polystyrene polymacromonomers in toluene [20,21], but it is quite unlikely to compensate for the small $\lambda_{\mathrm{b}}{ }^{-1}$. Thus, the present estimate of $\lambda^{-1}$ may be taken to show that the Nakamura-Norisuye theory is inapplicable to the polyelectrolyte brush. This is attributable to the fact that the long-range nature of electrostatic interactions is not considered in the theory. The high backbone stiffness of polyelectrolyte brushes provides an interesting theoretical problem.

\section{Conclusions}

We determined radii of gyration, particle scattering functions, and intrinsic viscosities for a series of polymacromonomer samples SF15 consisting of sodium poly(styrene sulfonate) (with a side-chain polymerization degree of 15) in $0.05 \mathrm{M}$ aqueous $\mathrm{NaCl}$ at $25^{\circ} \mathrm{C}$ and analyzed them on the basis of theories $[34,35,40]$ for the cylindrical wormlike chain. The theories consistently explain these data and yield mean values of $120 \mathrm{~nm}, 0.25$ $\mathrm{nm}$, and $5.5 \mathrm{~nm}$ for the Kuhn segment length, the contour length per main-chain residue, and the cylinder diameter, respectively. This Kuhn length is 7.5 times larger than that for the polystyrene polymacromonomer with the equivalent side-chain length in toluene, a good solvent. Electrostatic repulsions between charged groups of the polyelectrolyte brush are responsible for this backbone stiffening.

\section{Acknowledgments}

The synchrotron radiation experiments were performed at the BL40B2 in SPring-8 with the approval of the Japan Synchrotron Radiation Research Institute (JASRI) (Proposal No. 2007A1556).

\section{References}

[1] Decker PD. Makromol Chem 1969; 125: 136-160.

[2] Noda I, Horikawa T, Kato T, Fujimoto T, Nagasawa M. Macromolecules 1970; 3: 795-799.

[3] Candau F, Rempp P. Eur Polym J 1972; 8: 757-768.

[4] Strazielle C, Herz J. Eur Polym J 1977; 13: 223-233.

[5] Terao K, Farmer BS, Nakamura Y, Iatrou H, Hong K, Mays JW. Macromolecules 2005; 38: 1447-1450. 
[6] Tsukahara Y, Mizuno K, Segawa A, Yamashita Y. Macromolecules 1989; 22: 1546-1552.

[7] Tsukahara Y, Tsutsumi K, Yamashita Y, Shimada S. Macromolecules 1990; 23: 5201-5208.

[8] Barrales-Rienda JM, Galicia CR, Freire JJ, Horta A. Macromolecules 1983; 16: 940-945.

[9] Freire JJ, Barrales-Rienda JM, Galicia CR, Horta A. Macromolecules 1983; 16: 946-950.

[10] Wintermantel M, Schmidt M, Tsukahara Y, Kajiwara K, Kohjiya S. Macromol Rapid Commun 1994; 15: 279-284.

[11] Wintermantel M, Gerle M, Fischer K, Schmidt M, Wataoka I, Urakawa H, Kajiwara K, Tsukahara Y. Macromolecules 1996; 29: 978-983.

[12] Kawaguchi S, Akaike K, Zhang ZM, Matsumoto H, Ito K. Polym J 1998; 30: 1004-1007.

[13] Terao K, Takeo Y, Tazaki M, Nakamura Y, Norisuye T. Polym J 1999; 31: 193-198.

[14] Terao K, Nakamura Y, Norisuye T. Macromolecules 1999; 32: 711-716.

[15] Terao K, Hokajo T, Nakamura Y, Norisuye T. Macromolecules 1999; 32: 3690-3694.

[16] Hokajo T, Terao K, Nakamura Y, Norisuye T. Polym J 2001; 33: 481-485.

[17] Fischer K, Schmidt M. Macromol Rapid Commun 2001; 22: 787-791.

[18] Hokajo T, Hanaoka Y, Nakamura Y, Norisuye T. Polym J 2005; 37: 529-534.

[19] Zhang B, Gröhn F, Pedersen JS, Fischer K, Schmidt M. Macromolecules 2006; 39: 8440-8450.

[20] Sugiyama M, Nakamura Y, Norisuye T. Polym J 2008; 40: 109-115.

[21] Nakamura Y, Norisuye T. Polym J 2001; 33: 874-878.

[22] Rühe J, Ballauff M, Biesalski M, Dziezok P, Gröhn F, Johannsmann D, Houbenov N, Hugenberg N, Konradi R, Minko S, Motornov M, Netz RR, Schmidt M, Seidel C, Stamm M, Stephan T, Usov D, Zhang H. Adv Polym Sci 2004; 165: 79-150.

[23] Takahashi A, Kato T, Nagasawa M. J Phys Chem 1967; 71: 2001-2010.

[24] Hirose E, Iwamoto Y, Norisuye T. Macromolecules 1999; 32: 8629-8634.

[25] Iwamoto Y, Hirose E, Norisuye T. Polym J 2000; 32: 428-434.

[26] Yashiro J, Norisuye T. J Polym Sci B Polym Phys 2002; 40: 2728-2735.

[27] Kratky O, Porod G. Recl Trav Chim Pays-Bas 1949; 68: 1106.

[28] Vink H. Macromol Chem 1981; 182: 279-281.

[29] Berry GC. J Chem Phys 1966; 44: 4550-4564.

[30] Sato T, Norisuye T, Fujita H. Macromolecules 1983; 16: 185-189. 
[31] Norisuye T, Yanaki T, Fujita H. J Polym Sci Polym Phys Ed 1980; 18: 547-558.

[32] Amitani K, Terao K, Nakamura Y, Norisuye T. Polym J 2005; 37: 324-331.

[33] Nakamura Y, Sugiyama M, Amitani K, Norisuye T. Polym J 2007; 39: 1098-1104.

[34] Benoit H, Doty P. J Phys Chem 1953; 57: 958-963.

[35] Nakamura Y, Norisuye T. J Polym Sci B 2004; 42: 1398-1407.

[36] Konishi T, Yoshizaki T, Saito T, Einaga Y, Yamakawa H. Macromolecules 1990; 23: 290-297.

[37] Yamakawa H, Stockmayer WH. J Chem Phys 1972; 57: 2843-2854.

[38] Shimada J, Yamakawa H. J Chem Phys 1986; 85: 591-599.

[39] Yamakawa H. Helical wormlike chains in polymer solutions. Berlin: Springer, 1997.

[40] Yamakawa H, Yoshizaki T. Macromolecules 1980; 13: 633-643.

[41] Yoshizaki T, Yamakawa H. J Chem Phys 1980; 72: 57-69. 
Table I. Molecular characteristics of polymacromonomer consisting of Na poly(styrene sulfonate) in $0.05 \mathrm{M}$ aqueous $\mathrm{NaCl}$ at $25^{\circ} \mathrm{C}$

\begin{tabular}{|c|c|c|c|c|c|}
\hline Sample & $10^{-5} M_{\mathrm{w}}$ & $\begin{array}{l}\left\langle S^{2}\right\rangle_{\mathrm{z}}{ }^{1 / 2} \\
(\mathrm{~nm})\end{array}$ & $\begin{array}{l}10^{5} A_{2} \\
\left(\mathrm{~mol} \mathrm{~cm}^{3} \mathrm{~g}^{-2}\right)\end{array}$ & {$[\eta]\left(\mathrm{cm}^{3} \mathrm{~g}^{-1}\right)$} & $k^{\prime}$ \\
\hline SF15-1 & 71 & 104 & 4.4 & $170\left(230^{b}\right)$ & $0.3\left(0.5^{b}\right)$ \\
\hline SF15-2 & 68 & 97 & 3.7 & $118\left(191^{b}\right)$ & $0.5\left(0.6^{b}\right)$ \\
\hline SF15-3 & 35.5 & 54 & 4.8 & 59 & 0.6 \\
\hline SF15-4 & 29.5 & 57 & 9.2 & & \\
\hline SF15-5 & 13.6 & 34.5 & 25 & 17.0 & 0.5 \\
\hline SF15-6 & 1.36 & & 24 & 6.1 & 3 \\
\hline SF15-7 & 1.10 & 3.55 & 24 & 4.3 & 5 \\
\hline SF15-8 & 0.583 & 2.70 & 54 & 4.3 & 5 \\
\hline SF15-9 & $0.220\left(0.26^{\mathrm{a}}\right)$ & 2.23 & 180 & 6.0 & 3 \\
\hline
\end{tabular}

${ }^{\mathrm{a}} 10^{-5} \mathrm{M}_{\mathrm{z}}{ }^{\mathrm{b}}$ Data in $0.005 \mathrm{M}$ aqueous $\mathrm{NaCl}$ at $25^{\circ} \mathrm{C}$. 


\section{FIGURE CAPTIONS}

Figure 1. Repeating unit of a polymacromonomer consisting of sodium poly(styrene sulfonate). $\quad N$ and $n$ denote the number of main chain residues and that of side chain residues, respectively.

Figure 2. Berry plots for indicated samples of the SF15 polymacromonomer in $0.05 \mathrm{M}$ aqueous $\mathrm{NaCl}$ at $25^{\circ} \mathrm{C}$. (a) $\lambda_{0}=436 \mathrm{~nm}$ (b) $\lambda_{0}=546 \mathrm{~nm}$.

Figure 3. Guinier plots for indicated SF15 samples in $0.05 \mathrm{M}$ aqueous $\mathrm{NaCl}$ at $25{ }^{\circ} \mathrm{C}$.

Figure 4. $N_{\mathrm{w}}$-dependence of $\left\langle\mathrm{S}^{2}\right\rangle_{\mathrm{z}}{ }^{1 / 2}$ for $\mathrm{SF} 15$ in $0.05 \mathrm{M}$ aqueous $\mathrm{NaCl}$ at $25^{\circ} \mathrm{C}$ (circles) and that for polystyrene polymacromonomer F15 in toluene at $15^{\circ} \mathrm{C}$ (triangles, [14,33]) and in cyclohexane at $34.5^{\circ} \mathrm{C}$ (squares, [14,32]).

Figure 5. Concentration dependence of $\left(\ln \eta_{\mathrm{r}}\right) / c$ for low molecular weight samples of SF15 in (a) $0.005 \mathrm{M}$ and (b) $0.05 \mathrm{M}$ aqueous $\mathrm{NaCl}$ at $25^{\circ} \mathrm{C}$. Open circles, SF15-6; filled circles, SF15-7; open triangles, SF15-8; filled triangles, SF15-9.

Figure 6. $N_{\mathrm{w}}$-dependence of $[\eta] M_{0}$ for SF15 in $0.05 \mathrm{M}$ aqueous $\mathrm{NaCl}$ at $25{ }^{\circ} \mathrm{C}$ (open circles) and in $0.005 \mathrm{M}$ aqueous $\mathrm{NaCl}$ at $25^{\circ} \mathrm{C}$ (filled circles). Triangles and squares represent our previous data [15] for F15 in toluene at $15^{\circ} \mathrm{C}$ and in cyclohexane at $34.5^{\circ} \mathrm{C}$, respectively.

Figure 7. (a) Comparison between the experimental $\left\langle S^{2}\right\rangle_{\mathrm{Z}}^{1 / 2}$ (circles) for SF15 in $0.05 \mathrm{M}$ aqueous $\mathrm{NaCl}$ at $25^{\circ} \mathrm{C}$ and the theoretical curve calculated from eq 1 with eq 2 for the cylindrical wormlike chain with $M_{\mathrm{L}}=12000 \mathrm{~nm}^{-1}, \lambda^{-1}=130 \mathrm{~nm}, d=5.5 \mathrm{~nm}$, and $\delta=1.5$ nm. (b) Comparison for [ $\eta]$. The curve is calculated from eq 3 with $M_{\mathrm{L}}=14000 \mathrm{~nm}^{-1}$, $\lambda^{-1}=110 \mathrm{~nm}, d=5.5 \mathrm{~nm}$, and $\delta=4.3 \mathrm{~nm}$.

Figure 8. Kratky plots for (a) SF15-7, (b) SF15-8, and (c) SF15-9 in 0.05 M aqueous $\mathrm{NaCl}$ at $25^{\circ} \mathrm{C}$. Solid curves, theoretical values for the wormlike cylinder with $M_{\mathrm{L}}=$ $12000 \mathrm{~nm}^{-1}, \lambda^{-1}=130 \mathrm{~nm}, d=5.5 \mathrm{~nm}$, and $\delta=1.5 \mathrm{~nm}$. 


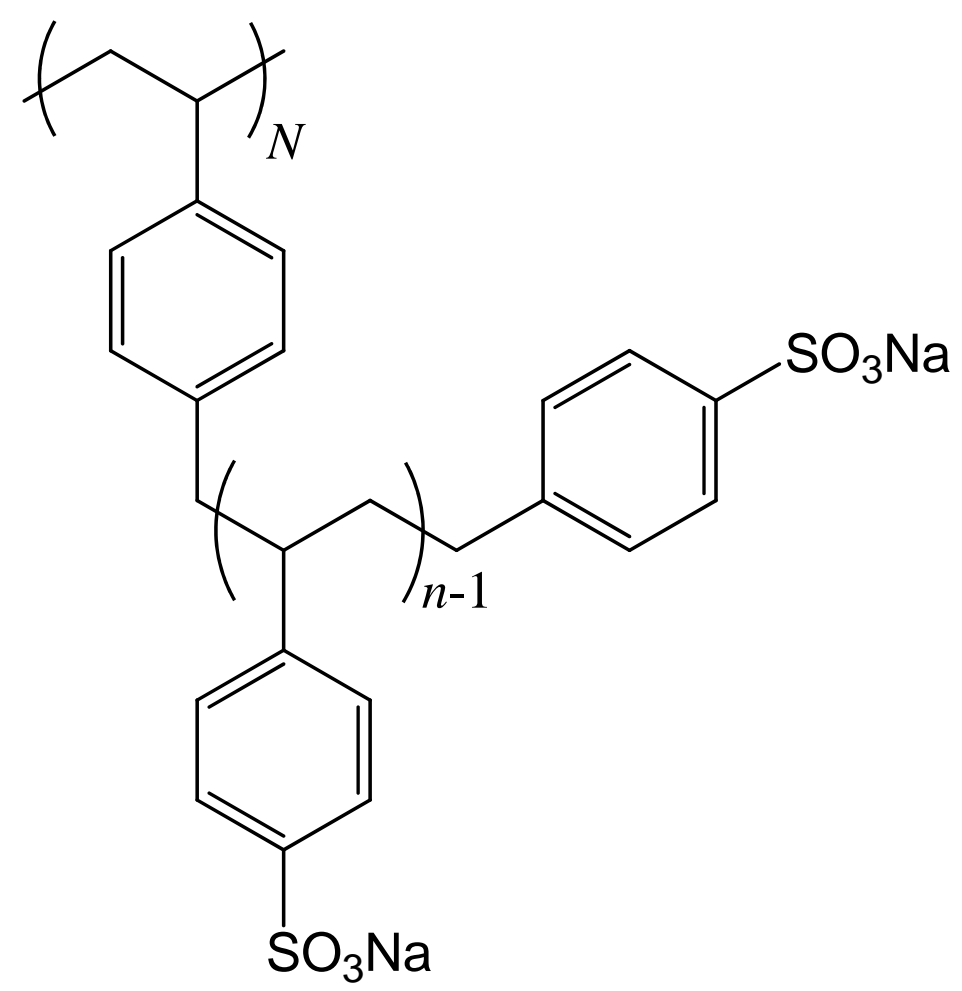

Figure 1. Kanemaru et al. 


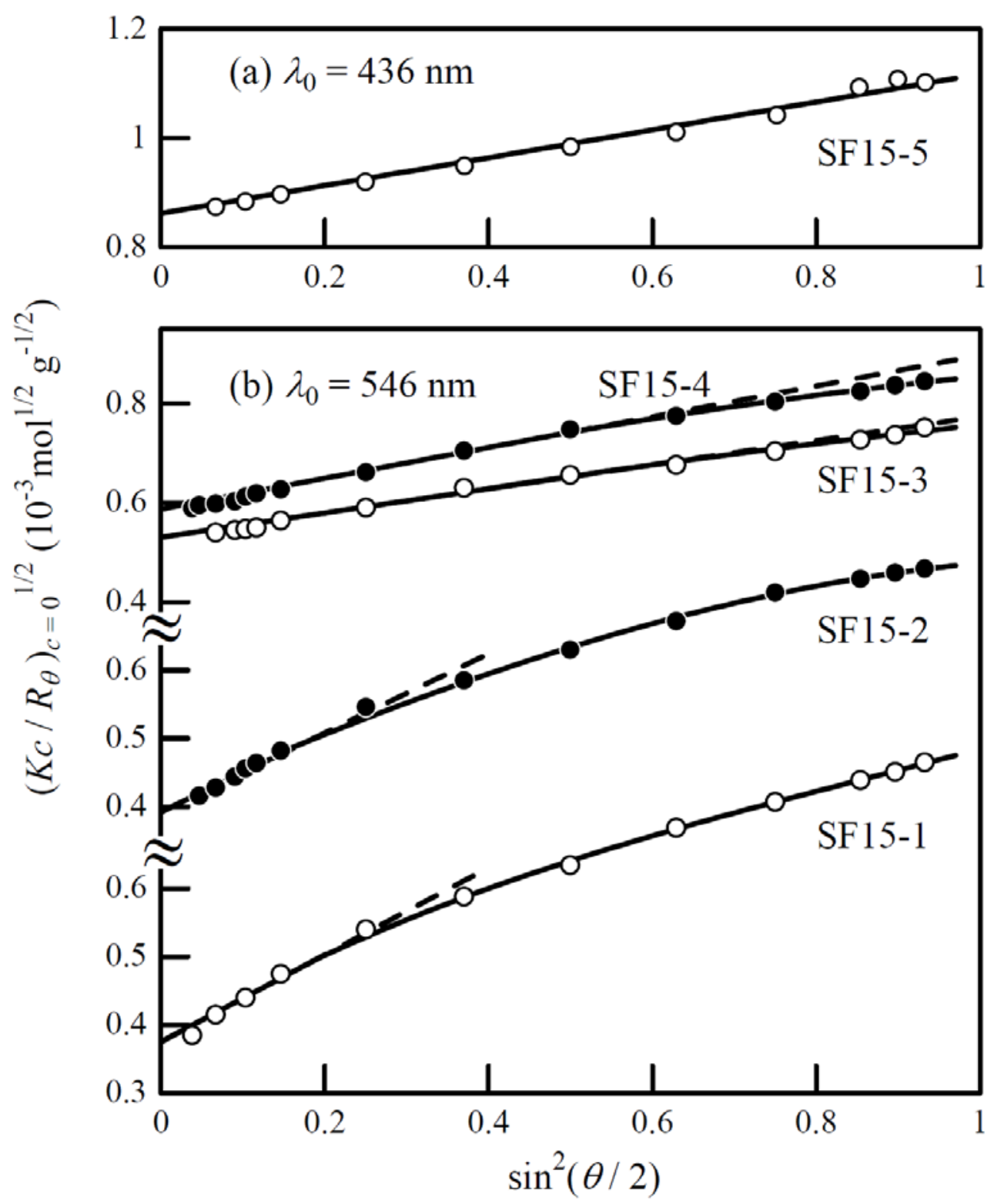

Figure 2. Kanemaru et al. 


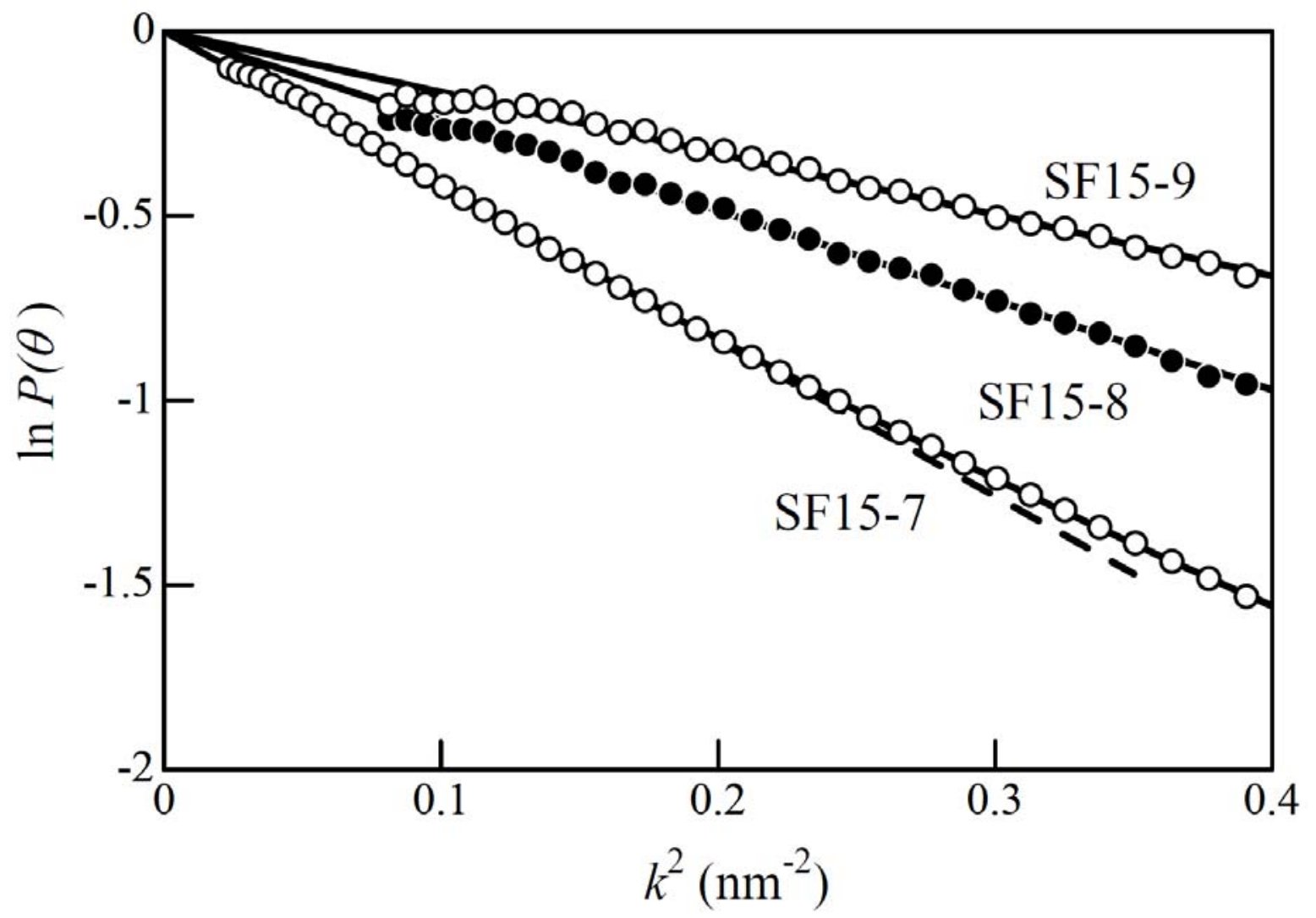

Figure 3. Kanemaru et al. 


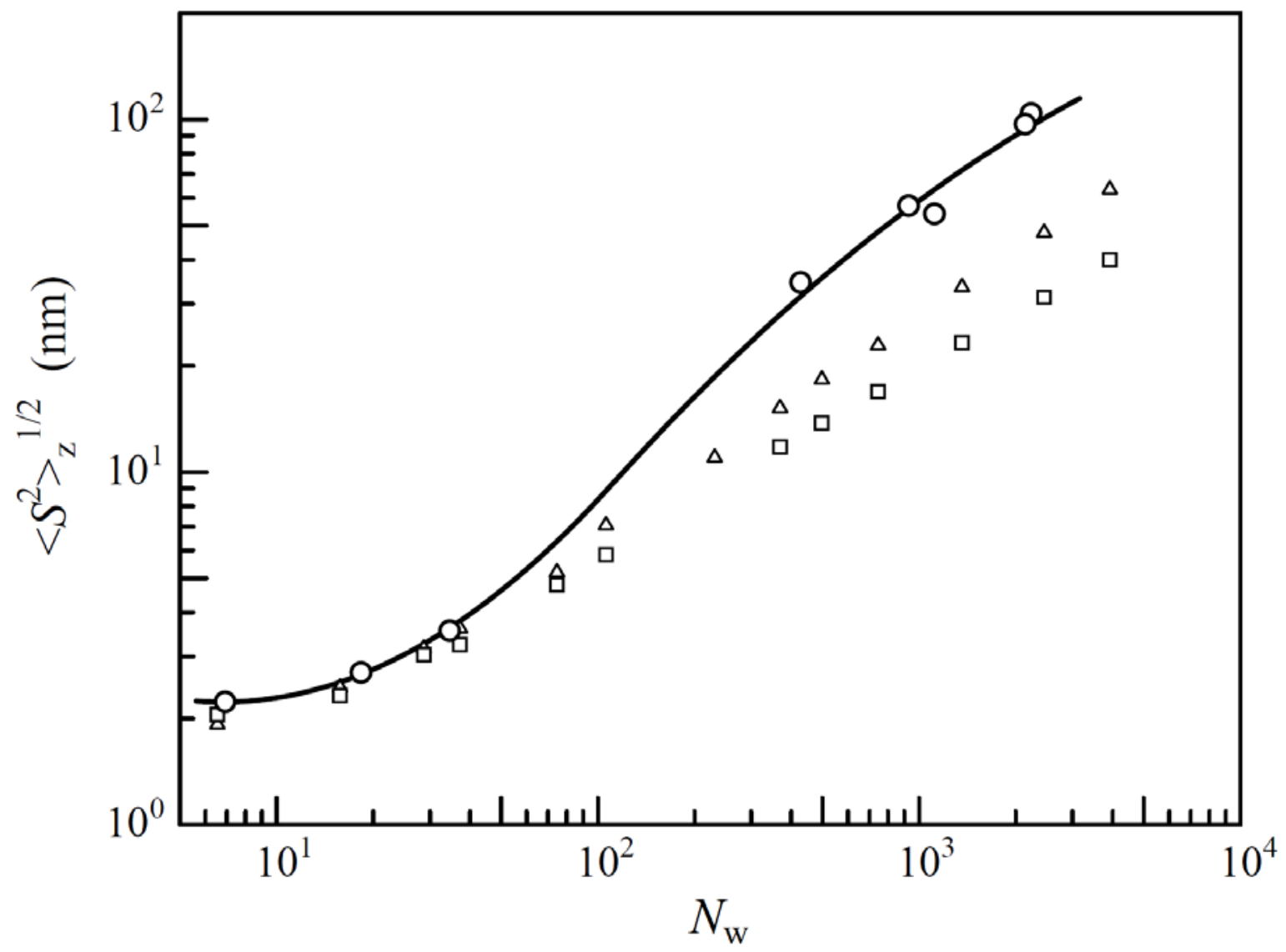

Figure 4. Kanemaru et al. 


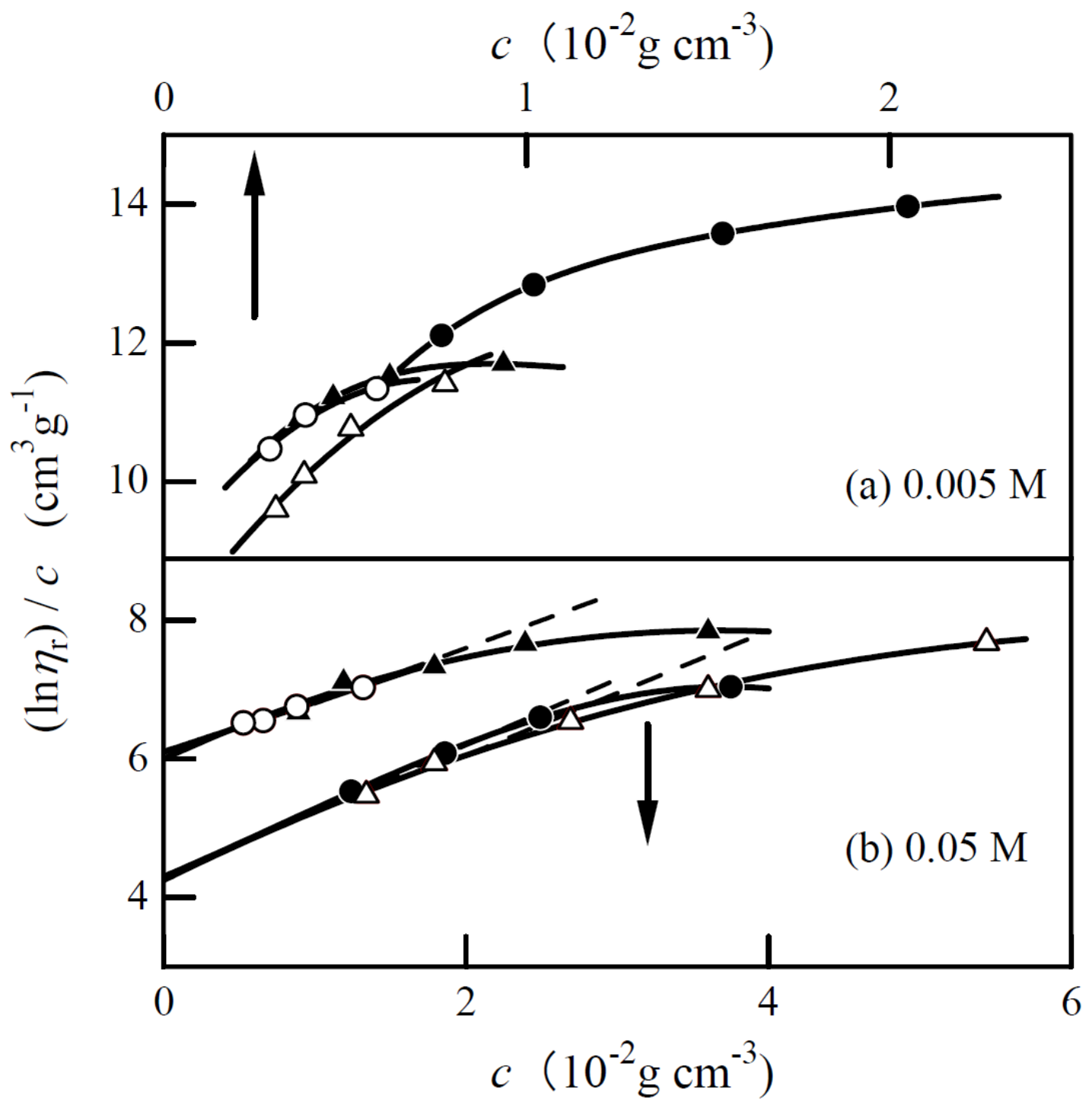

Figure 5. Kanemaru et al. 


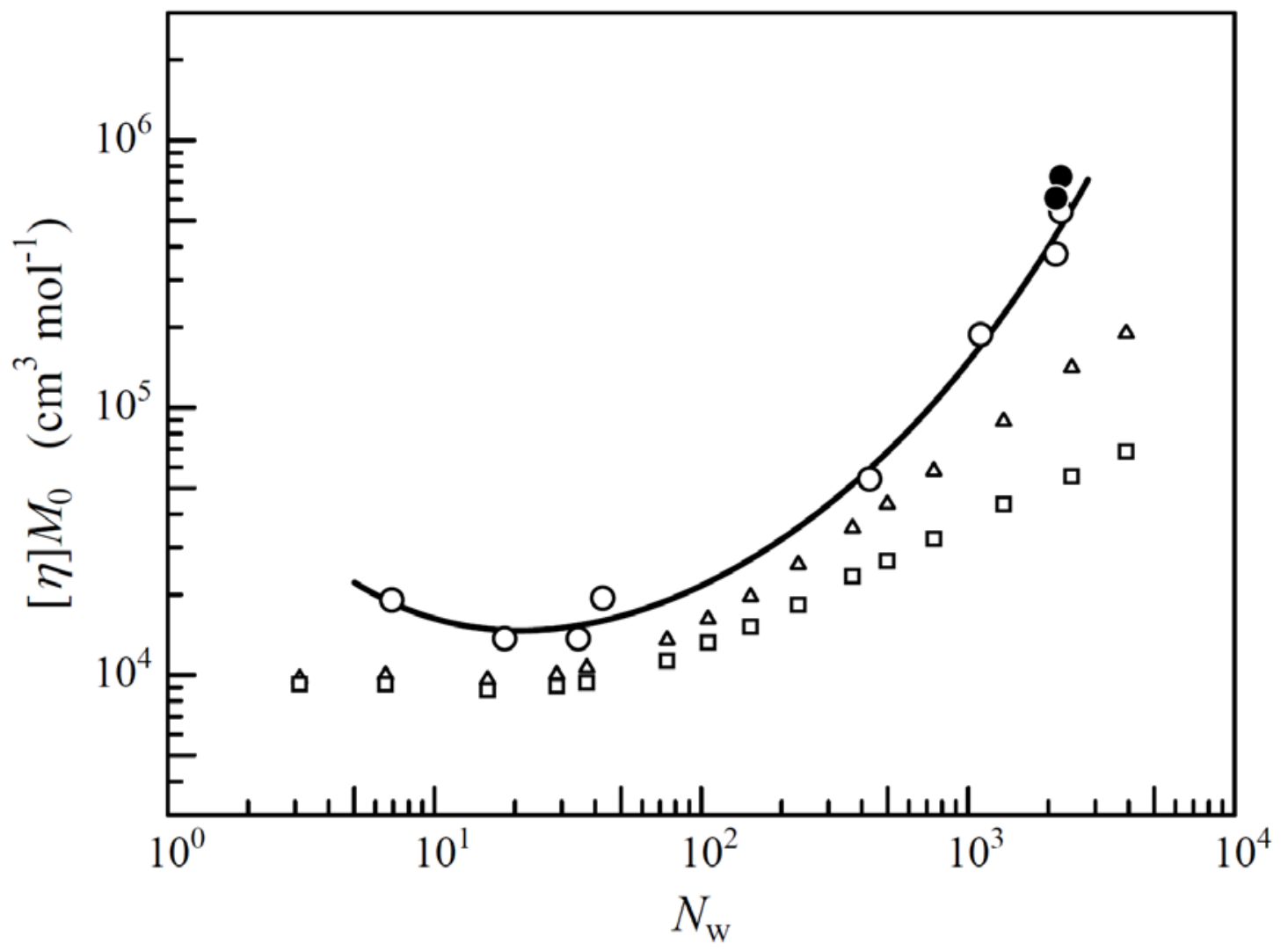

Figure 6. Kanemaru et al. 

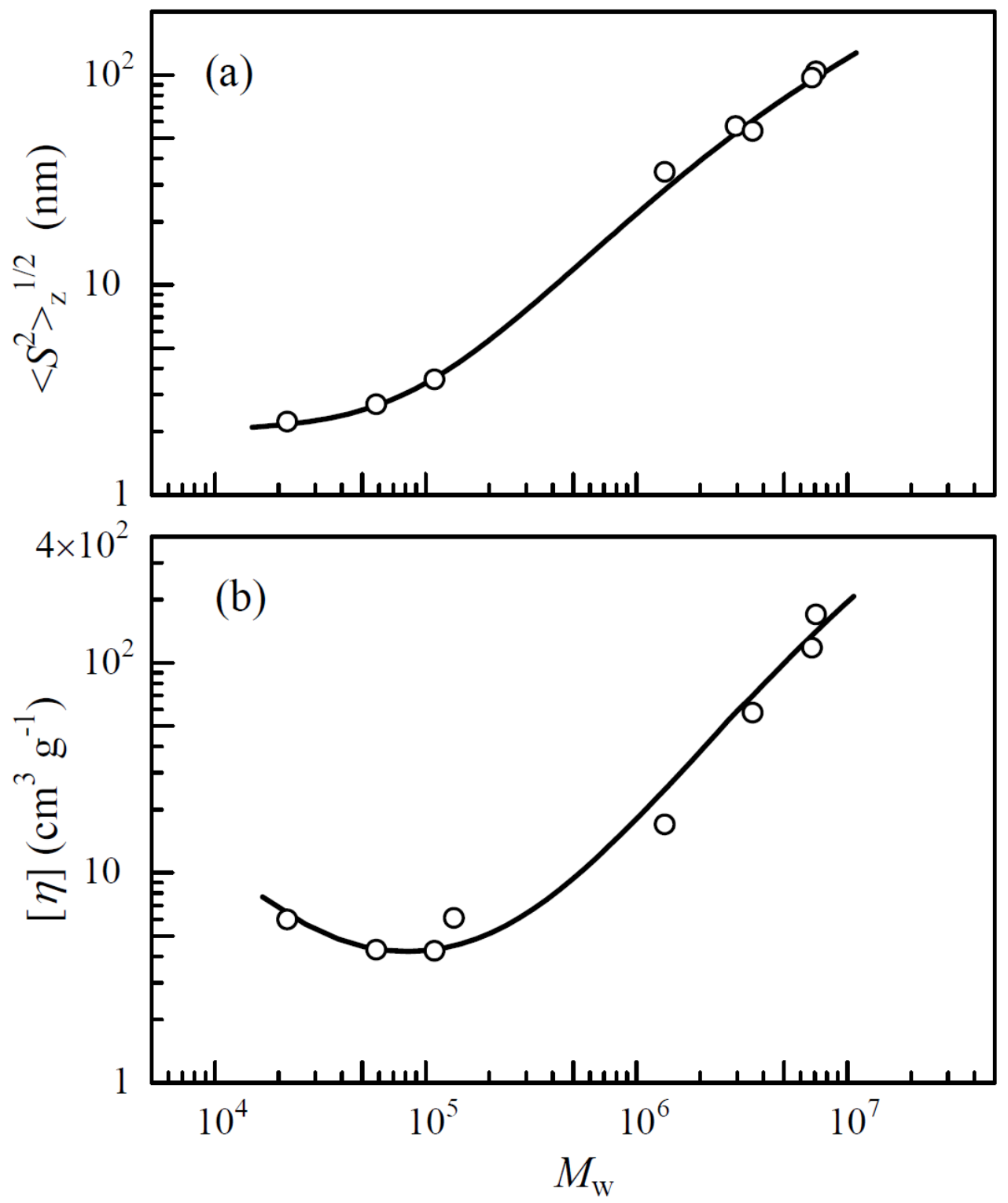

Figure 7. Kanemaru et al. 

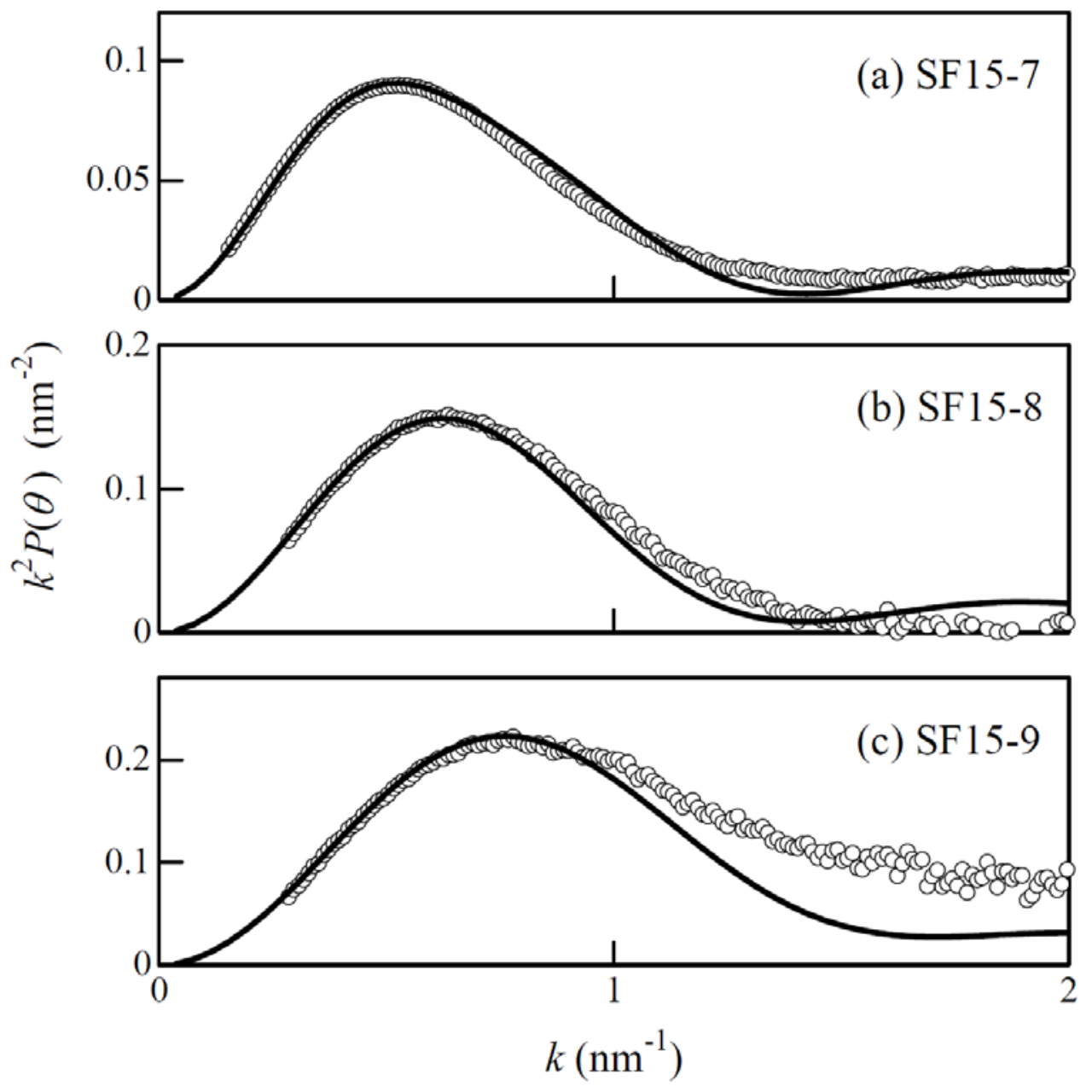

Figure 8. Kanemaru et al. 